\title{
Medizinberufe im Wandel
}

\section{Matthias Scholer}

Chefredaktor Schweizerische Ärztezeitung

Der Fachkräftemangel stellt insbesondere die Versorgung chronisch Kranker und multimorbider Patienten vor grosse Herausforderungen. Ein zentraler Lösungsansatz ist die Integration neuer Berufsgruppen. Auf dem Papier stehen die Konzepte für diese neuen Gesundheitsberufe bereits seit einiger Zeit. Doch wie weit ist die Integration im Alltag fortgeschritten, und welche Hindernisse verlangsamen die Prozesse? Wir haben bei drei Gesundheitsprofis nachgefragt und sie aus ihrem Alltag berichten lassen.

Caroline Fröhli: «Das neue Berufsbild MPK und die damit verbundenen Möglichkeiten ist bei den Ärztinnen und Ärzten noch zu wenig bekannt»

«Der Berufsalltag als Medizinische Praxisassistentin (MPA) gefiel mir schon immer. Mein Wissen erweitern und im Alltag neue Aufgaben übernehmen sind Herausforderungen, denen ich mich schon immer gerne stellte. Als dann die neue Weiterbildung zur Medizinischen Praxiskoordinatorin (MPK) angeboten wurde, zögerte ich nicht lange. Ich absolvierte beide möglichen Ausrichtungen einer MPK: die praxisleitende und die klinische Richtung.

Heute arbeite ich als MPA/MPK-Botschafterin bei der Argomed Ärzte AG. In dieser Funktion habe ich die Aufgabe, ein Netzwerk für engagierte MPA/MPK für praxisrelevante Themen aufzubauen, zu pflegen und

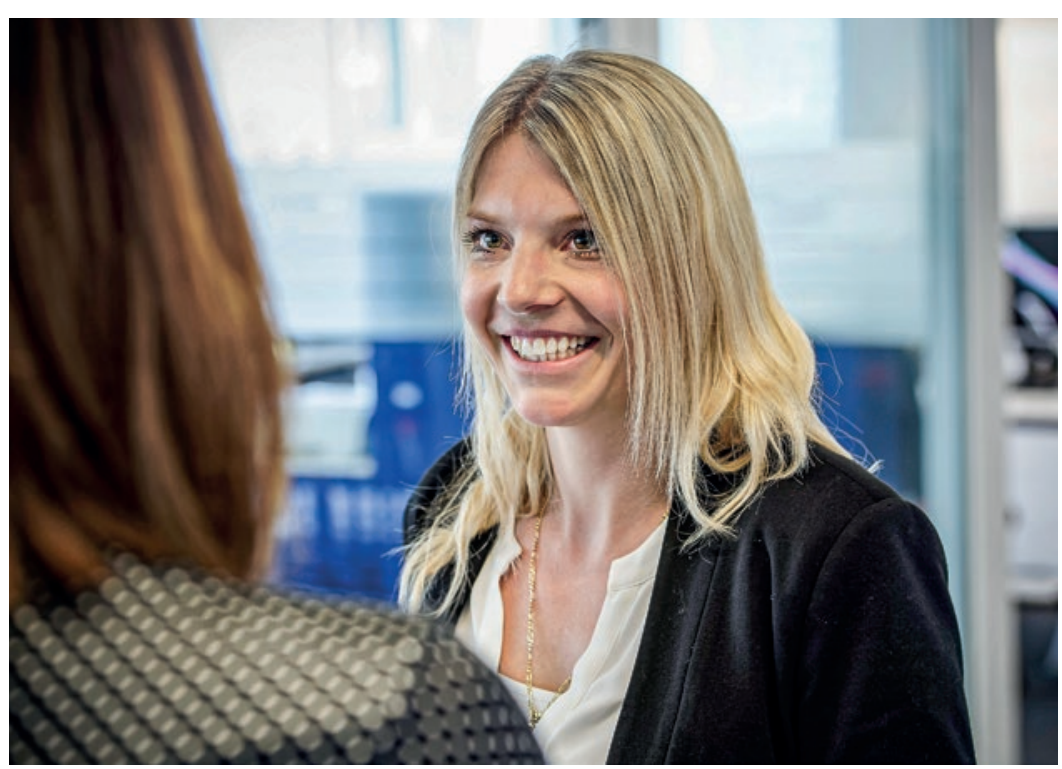

Caroline Fröhli setzt sich aktiv für die Stärkung der Rolle MPA/MPK ein. gezielt die Rolle als MPA/MPK zu fördern und zu stärken. Zusätzlich zu dieser spannenden Aufgabe arbeite ich Teilzeit als MPA/MPK in einer Hausarztpraxis. Dort erledige ich alltägliche Arbeiten als MPA und habe nebenbei eine Sprechstunde für rheumatische Erkrankungen mit Vertiefung Osteoporose aufgebaut.

Bei dieser MPK-Sprechstunde berate und begleite ich Patientinnen und Patienten mit folgenden drei Zielen: Erstens die Klärung offener Fragen oder bei einer Neudiagnose eine adäquate Aufklärung der Patientinnen und Patienten über ihre chronische Krankheit. Häufig war es den Betroffenen während der vorangegangenen Sprechstunde nicht möglich, alle Fragen zu stellen, die ihnen am Herzen liegen, oder sie sind sich nicht sicher, ob sie alles richtig verstanden haben. Das zweite Ziel ist die Förderung des Selbstmanagements. Dabei geht es unter anderem um Aspekte, wie eine Patientin, ein $\mathrm{Pa}$ tient krankheitsbezogene Warnsignale frühzeitig erkennen kann und lernt, bei welchen Anzeichen möglichst rasch eine Arztpraxis aufgesucht werden sollte. Es hat sich gezeigt, dass chronisch kranke Patienten häufig zu lange warten, bis sie ärztliche Hilfe in Anspruch nehmen, und somit eine stationäre Behandlung unausweichlich ist. Drittens führe ich eine Medikamentenkontrolle durch. Werden die verschriebenen Arzneimittel korrekt eingenommen? Bestehen Unklarheiten oder Ängste in Bezug auf mögliche Nebenwirkungen? Immer mit dem übergeordneten Ziel, die Adhärenz der Therapie möglichst hochzuhalten.

Während meiner Sprechstunde ist der behandelnde Arzt unserer Praxis je nach Bedarf ebenfalls anwesend, um gemeinsam die Laborwerte zu besprechen oder 
zum Beispiel eine neue Dosierung eines Medikamentes anzupassen. Dies gehört selbstverständlich nach wie vor in den Aufgabenbereich des Arztes.

Unsere Rheumapatienten und -patientinnen begrüssen das Angebot der MPK-Sprechstunde sehr. Vor allem wird die individuelle Betreuung, der persönliche Austausch und die zur Verfügung gestellte zeitliche Ressource sehr geschätzt.

Leider bestehen in der Umsetzung noch einige Stolpersteine, primär aus regulatorischer Sicht. So ist z.B. die Finanzierung von MPK-Leistungen noch ungenügend geregelt. Erst im Diabetesbereich gibt es für die Abrechnung eine TARMED-Position. Meine Arbeit mit den Rheumapatientinnen und -patienten wird von der Rheumaliga Schweiz über das Projekt 'Kompass' mitfinanziert. Auch besteht noch viel Aufklärungsbedarf: Die unklare Abgeltung und der Ressourcenbedarf, der beim Einsatz einer klinischen MPK entsteht, schrecken bislang viele Praxen ab. Das neue Berufsbild MPK und die damit verbundenen Möglichkeiten sind bei den Ärztinnen und Ärzten noch zu wenig bekannt. Arzt, Ärztin, MPA und MPK sollten ein Team mit klar defi- nierten Kompetenzen und Schnittstellen bilden, denn so kann das Ressourcenproblem in der Hausarztpraxis entschärft werden. Dank der Delegation gezielter Betreuungsaufgaben an die dafür ausgebildeten MPA/ MPK erhält die Ärzteschaft freie Kapazitäten, um weitere Patienten zu betreuen oder andere Aufgaben wahrzunehmen.»

Caroline Fröhli, Medizinische Praxisassistentin (MPA)/ Medizinische Praxiskoordinatorin (MPK) in der Arztpraxis St.Wolfgang und MPA/MPK-Botschafterin bei der Argomed Ärzte AG

MPK: Medizinische Praxisassistentinnen (MPA) haben die Möglichkeit, sich zur Medizinischen Praxiskoordinatorin (MPK) weiterzubilden, wobei zwischen einer praxisleitenden und einer $\mathrm{kli}$ nischen Ausrichtung unterschieden wird. MPK, die sich für eine klinische Weiterbildung entscheiden, erhalten vertiefte Einblicke in ausgewählte Krankheitsbilder wie Diabetes, COPD oder Wundversorgung. Sie können danach die Beratung und Kontrolle der Langzeitversorgung von stabilen chronisch Kranken innerhalb des Praxissettings übernehmen.

\section{Simone Beck: «Als Klinische Fachspezialistin bin ich Teil des ärztlichen Teams»}

«Ich schloss vor etwas mehr als neun Jahren meine Ausbildung zur Physiotherapeutin ab. Als vor bald zwei Jahren eine Stelle für eine Klinische Fachspezialistin hier an der ZurzachCare Rehaklinik Sonnmatt intern ausgeschrieben wurde, habe ich mich erfolgreich darauf beworben und an der ZHAW berufsbegleitend den

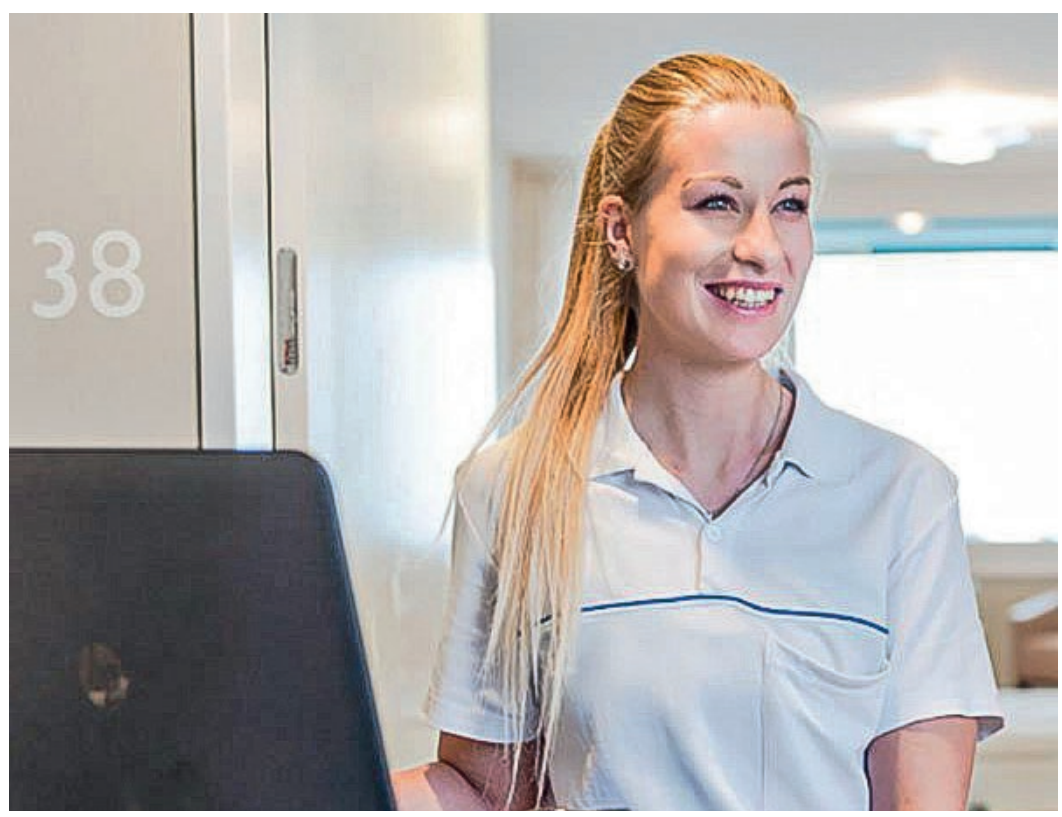

Interprofessionalität gehört zum Alltag der Klinischen Fachspezialistin Simone Beck.
Ausbildungsgang 'CAS Klinische Fachspezialistin/Klinischer Fachspezialist' absolviert. Zwischenzeitlich arbeiten bei uns im Haus nun insgesamt vier im englischen Sprachgebrauch als Physician Assistant bezeichnete Kolleginnen und Kollegen. Da mir diese Stelle grosse Freude bereitet und ich viel von der breiten Ausbildung profitieren kann, habe ich beschlossen, den Studiengang nun bis zum Abschluss mit einem Master of Advanced Studies (MAS) fortzuführen.

Als Klinische Fachspezialistin bin ich Teil des ärztlichen Teams. Dieses besteht in der Rehaklinik aus dem Chefarzt, zwei Oberärzten und uns vier Klinischen Fachspezialistinnen. Wenn ich Frühdienst habe, beginnt mein typischer Arbeitstag um sieben Uhr mit der Übergabe des Telefons des Arztes, der Ärztin, die Nachtdienst hatte. Sie rapportieren mir dabei allfällige Vorkommnisse, die für uns relevant sind. Die nächste Stunde ist dann für Notfälle, Fragen seitens der Pflege, der administrativen Bearbeitung der aktuellen Einund Austritte und das Erstellen und Einreichen von Kostengutsprachen reserviert.

Um 8.15 Uhr findet der Ärzterapport statt. Während diesem berichte ich über die Vorkommnisse während der Nacht und stelle den Ärztinnen und Ärzten die geplanten Eintritte vor. Danach kümmere ich mich um die Austritte. Ich bringe den Patientinnen und Patien- 
ten die Austrittsberichte, Verschreibungen und Verordnungen und erkläre ihnen diese im Rahmen eines Austrittsgesprächs.

Um 9 Uhr steht dann die Visite an. Zwei Mal in der Woche mache ich diese alleine. Falls sich dabei medizinische Fragen ergeben, bespreche ich mich nachträglich mit dem Oberarzt. An den Tagen, an denen auch die Ärztinnen und Ärzte mit auf Visite kommen, stelle ich die Patientinnen und Patienten vor und aktualisiere im Nachgang die Patientendokumentation.

Danach kümmere ich mich um die Eintritte. Dazu gehört neben dem Anamnesegespräch und der Evaluation allfälliger Nebenerkrankungen auch eine körperliche Untersuchung. Die Befunde präsentiere ich der Oberärztin, bevor wir den Patienten zusammen besuchen, um allfällige offene Fragen zu klären.

Einmal in der Woche gibt es einen interdisziplinären Rapport mit Vertreterinnen der Physiotherapie, der Pflege, dem Sozialdienst, einer Neuropsychologin und uns vom ärztlichen Dienst. Dabei besprechen wir spezielle Fälle, bei denen eine interdisziplinäre Betreuung während und nach dem Klinikaufenthalt notwendig ist. Für eine gute Patientenbetreuung braucht es schliesslich die Zusammenarbeit aller Disziplinen.

Als Klinische Fachspezialistin sorge ich für einen reibungslosen Ablauf vom Eintritt bis zum Austritt und stehe den Patientinnen und Patienten als Ansprechpartnerin für sämtliche Fragen rund um ihr Wohlerge- hen zur Verfügung. Dass diese Dienstleistung auch vonseiten der Kranken sehr geschätzt wird, zeigt sich in den positiven Rückmeldungen im Rahmen der Patientenbefragungen.

Ich bin überzeugt, dass die Funktion einer Klinischen Fachspezialistin, eines Klinischen Fachspezialisten in Zukunft noch an Bedeutung gewinnen wird, damit die Kommunikation zwischen allen Beteiligten optimal läuft und schnell und effizient reagiert werden kann. Persönlich wünsche ich mir, dass der Beruf künftig dank einem Studiengang bis zur Bachelorstufe auch Interessierten ohne medizinische Vorkenntnisse offensteht und unser Berufsbild rasch an Bekanntheit gewinnt.»

\section{Simone Beck, Klinische Fachspezialistin, ZurzachCare Rehaklinik Sonnmatt, Luzern}

\section{Michaela Moser: «Die Vielschichtigkeit meines Berufes hat auch seine} Schattenseiten»

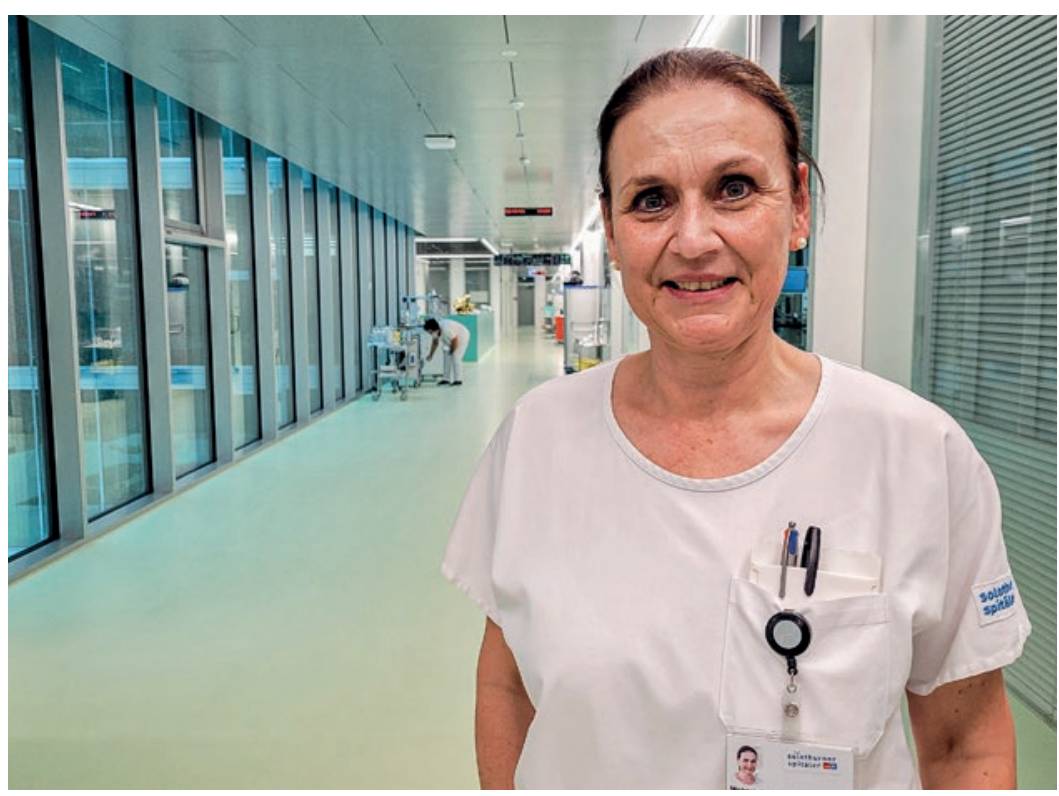

Die Pflegeexpertin APN CNS Michaela Moser liebt die Vielschichtigkeit ihres Berufes.
«Ich bin seit rund 30 Jahren in der Intensivpflege tätig. Nachdem meine Kinder gross genug waren, entschied ich mich, mein Arbeitspensum wieder zu erhöhen und mein Fachwissen zu vertiefen. So absolvierte ich die Ausbildung zur Erwachsenenbildnerin und war danach während zwölf Jahren als Berufsbildnerin im Bereich der Intensivpflege tätig. Als auf unserer Abteilung eine Pflegeexpertin zu arbeiten begann, welche im Ausland ein Masterstudium der Pflegewissenschaften abgeschlossen hatte, war dies für mich der nächste Wendepunkt. Denn wann immer ich mit dieser Kollegin sprach, diskutierten wir wissenschaftlich fundiert über pflegerische Themen. Eine für mich ganz neue und spannende Dimension. Ich entschloss mich deshalb, im Alter von 47 Jahren ein Pflegestudium mit Masterabschluss zu absolvieren. Diesen Entscheid habe ich nie bereut. Ich gab folglich sogar meine Stelle im Bereich der Berufsbildung zugunsten einer Stelle als Pflegeexpertin 
APN CNS auf. Seit fünf Jahren bin ich nun für zwei Intensivstationen zuständig.

Ein Charakteristikum meines Alltags ist, dass man einen sehr hohen Grad an Flexibilität braucht. Am Morgen weiss ich nie, in welchem Bereich meine Expertise am meisten gebraucht wird. Meine Aufgaben umfassen einerseits die Unterstützung der Teams und andererseits Entwicklungsarbeit. An Tagen, an denen ich mich mehr um konzeptionelle Arbeiten kümmere, bin ich trotzdem sicher einmal auf der Station anzutreffen, um mir einen Überblick über die Patientinnen und Patienten auf unserer Abteilung zu verschaffen und um den Pflegenden und der Ärzteschaft die Möglichkeit zu geben, sich mit mir auszutauschen.

Die Ärztinnen und Ärzte kommen häufig auf mich zu, wenn es darum geht, komplexere Fälle auf eine andere Abteilung zu verlegen oder einen Reha-Platz zu suchen. Dabei bilde ich eine Schnittstelle zwischen Intensivstation und den übernehmenden Stationen oder Institutionen.

Die Pflegenden wiederum kommen mit den unterschiedlichsten Themen zu mir. Das können pflegerische oder ethische Fragestellungen sein, aber auch Fragen im Umgang mit Angehörigen. Gemeinsam eruieren wir dann, ob ich eine Aufgabe direkt übernehme oder ob ich die Pflegenden nur berate. Meine Aufgaben reichen somit von Routinearbeiten am Patientenbett bis hin zum Coaching der Teams.

Die Vielschichtigkeit meines Berufes hat auch ihre Schattenseiten. Da dieses Berufsbild in der Schweiz noch wenig bekannt ist, fehlt vielen Pflegenden sowie Ärztinnen und Ärzten das nötige Rollenverständnis, um die Ressourcen einer Pflegeexpertin APN CNS effizient zu nutzen. Wenn ich mit Personen, die im Ausland tätig waren, zusammenarbeite, funktioniert es auf Anhieb besser, da in vielen Ländern die Pflegeexpertin im stationären Bereich bereits gut implemen- tiert ist. Damit kennen diese Personen das Potenzial einer Pflegeexpertin APN CNS aus eigener Erfahrung und wissen es auszuschöpfen. In der Literatur sind die Aufgaben einer Pflegeexpertin, im Vergleich zum Beispiel zu einer Stationsleitung, eher auf einer Metaebene beschrieben. Deshalb lässt sich auch der direkte Output schwer messen, und es macht es zusätzlich schwierig, unsere Rolle zu erklären.

Interprofessionalität gehört seit langem zum Alltag auf Intensivstationen. Doch wie erfolgreich diese umgesetzt werden kann, hängt schliesslich von den daran beteiligten Menschen und deren Einstellung ab. Wenn man mit Personen zusammenarbeitet, die noch stark hierarchisch strukturiert sind, wird es schwierig. Somit funktioniert es einmal besser, einmal schlechter. Auf alle Fälle sind wir noch weit davon entfernt, sagen zu können, wir hätten eine einheitliche Kultur der Interprofessionalität auf den einzelnen Stationen.»

\section{Michaela Moser, Pflegeexpertin APN CNS,}

Solothurner Spitäler AG (Kantonsspital Olten und Bürgerspital Solothurn)

\section{Bildnachweis}

Alle Porträtfotos zVg

APN: Die Ausbildung zur Advanced Practice Nurse (APN) erfolgt über einen Master-Studiengang an einer Fachhochschule oder Universität. Die Grundausrichtung der Ausbildung ist angewandt-wissenschaftlich mit einer hohen Praxisorientierung. Ausgebildete APN planen in Zusammenarbeit mit den Ärztinnen und Ärzten Behandlungen und führen anspruchsvolle medizinische Massnahmen selbständig durch. Zudem leisten sie auf die Betroffenen zugeschnittene, evidenzbasierte Pflege, so dass der Alltag erfolgreich bewältigt werden kann.

Die Ausrichtung der Ausbildung zur APN kann mit verschiedenen Vertiefungsrichtungen auf den angestrebtenTätigkeitsbereich angepasst werden. Dazu zählen beispielsweise: Clinical Nurse Specialist (CNS), Forschung oder Nurse Practitioner (NP).

\section{Schwerpunktserie Interprofessionalität}

Die interprofessionelle Zusammenarbeit von Fachpersonen aus verschiedenen Gesundheitsberufen gilt als wichtiges Mittel, um den Herausforderungen im Gesundheitswesen zu begegnen. Aber wie weit ist die Schweiz in diesem Bereich tatsächlich? Welche Hürden und welche Chancen gibt es? In unserer Schwerpunktserie betrachten wir das Thema aus unterschiedlichen Perspektiven. 